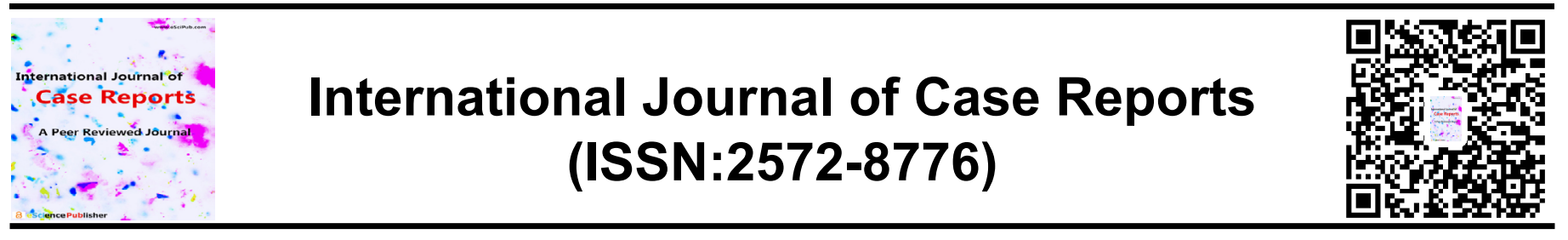

\title{
Extra-synovial Pigmented Villonodular Synovitis of the knee joint, Case Report
}

\author{
Rebar Mohammed Noori, Musaab Mohammed Hazim* \\ Department of Orthopedic Surgery, School of Medicine, University of Sulaimaniya, Iraq.
}

\begin{abstract}
Background: PVNS is a rare, benign \& aggressive disorder arising from either synovial joints or tendon sheaths; it may erode articular structures and bones. We present a case with unique features of PVNS being extra-synovial and by this report we open a gate for more researches in this field.
\end{abstract}

Case Presentation: This case report concerns a 35-year-old female with a history of right knee pain for 6-month duration proceeded by gradual swelling over posterior aspect of the knee, she denies any history of trauma, clinical examination was unremarkable but apart from tenderness over the infrapatellar region with full flexion.

MRI shows a heterogenous signal extra-articular and extrasynovial lesion in posterior aspect of the knee suggesting Pigmented Villonodular Synovitis, FNA revealed a hemosiderinladen macrophages and multinucleated giant cells, Tru-cut biopsy result was suggesting PVNS as synovial cells were seen admixed with hemosiderin-laden macrophages with fibroblastic elements.

Through posterior approach; the lesion was surgically excised and histopathological examination confirmed the diagnosis, the lesion was recurrent after 1-year and MRI revealed the same features, the lesion was excised by arthroscopic intervention.

Conclusion: We concluded that PVNS cannot be excluded when extra-synovial lesion is assessed, and further researches on this topic will expand our understanding of the etiological and pathological aspects of this tumor.

Keywords:Pigmented Villonodular Synovitis, Giant Cell Tumor, Knee, Synovitis, Benign tumor
*Correspondence to Author:

Dr. Musaab Mohammed Hazim

Department of Orthopedic Surgery, School of Medicine, University of Sulaimaniya, Iraq.

How to cite this article:

Rebar Mohammed Noori, Musaab Mohammed Hazim. Ex-trasynovial Pigmented Villonodular Synovitis of the knee joint, Case Report. International Journal of Case Reports, 2021; 5:192

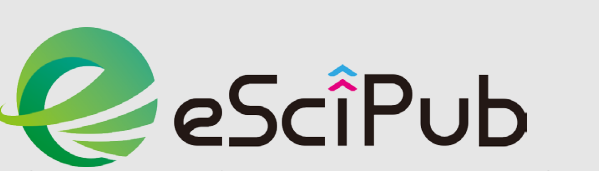
eSciPub LLC, Houston, TX USA. Website: http://escipub.com/ 


\section{Introduction}

PVNS is a rare, benign \& aggressive disorder arising from either synovial joints or tendon sheaths; it may erode articular structures and bones. [19]

It is a proliferative reactive (rather than neoplastic) synovial process characterized by mononuclear stromal cells, hemorrhage, histiocytes, and giant cells with exuberant proliferation of synovial villi and nodules. ${ }^{[16][18]}$ Highly vascular villi are lined with plump hyperplastic synovial cells, hemosiderin-stained multinucleated giant cells, and chronic inflammatory cells. ${ }^{[18]}$ It occurs mostly between 30 to 50 years old with equal gender distribution. [16]

Knee joint is affected most often ( $80 \%)$, followed in sequence by hip, shoulder and ankle but it may occur in any joint else. ${ }^{[16]}$

While $50 \%$ of patients report an earlier traumatic incident but still the exact etiology has not been known yet. ${ }^{16]}$

In recent years, some researchers have found that the chromosome morphology and number of PVNS are abnormal. The most distorted site was 1p11-13 on the short arm of the first chromosome [2], trisomy aberration of the seventh and the fifth chromosomes. ${ }^{[3]}$

PVNS represents a spectrum of "fibrohistocytic" disorders ranging from the most common giantcell tumor of tendon sheath (GCTTS) to localized and diffuse intra-articular lesions; the estimated incidence is 1.8 new cases annually per million populations [11], highlighting the rarity of this disorder. [19]

Simon described the localized type of the knee ${ }^{[13]}$ and Moser, in 1909, described the diffuse type of the disease ${ }^{[14]}$. Jaffe et al. proposed the term pigmented villonodular synovitis for these manifestations, but the nomenclature proposed by Granowitz defined that the term pigmented villonodular synovitis should be used for intraarticular lesions, pigmented villonodular bursitis for lesions located in the bursae and pigmented villonodular tenosynovitis for lesions originating from tendinous sheaths. ${ }^{[21]}$
The localized form is identical histologically to giant cell tumor of tendon sheath while the diffuse form also appears to be identical histologically to the localized form, but it involves the entire synovium [20], the diffuse form has a higher recurrence rate. Synovectomy is effective, but the recurrence rate is still high ( $8 \%$ $-56 \%)^{[1]}$

The patient usually presents with mono-articular pain and swelling, intra-articular localized lesions may cause mechanical symptoms [20] and may have a palpable mass while diffuse form may decrease joint range of motion. [16]

Extra-articular form (giant cell tumor of tendon sheath) usually affects the hand/wrist with a small, painless, superficial soft-tissue nodule. ${ }^{[16]}$ Recurrent atraumatic hemarthrosis is the hallmark, aspiration of the joint characteristically reveals serosanguineous or blood-tinged fluid, grossly it is reddish-brown stained synovium with extensive papillary projections, regarding histology; mononuclear stromal cell infiltrate within the synovium, and cells are round with a large nucleus and eosinophilic cytoplasm. ${ }^{16]}$

Hemosiderin-laden

macrophages, multinucleated giant cells, and foam cells present but these are not required for diagnosis, mitotic figures are relatively common. ${ }^{[16]}$

Children with an acute onset of swelling, pain, and refusal to bear weight frequently are subject to workup for septic arthritis, transient synovitis, or a bleeding disorder. More chronic joint symptoms receive a rheumatologic diagnosis, frequently juvenile idiopathic arthritis, with prolonged pharmacotherapy with minimal or transient symptomatic improvement. PVNS must be included in the differential of children and adolescents with acute or chronic hip pain that does not have a clear infectious origin or has failed to respond appropriately to pharmacotherapy and physical therapy. MRI with gradient echo sequences should be performed early to evaluate for this disease process. Failure to consider PVNS as a differential diagnosis may result in a delayed diagnosis, which may lead to progressive 
functional impairment and degenerative changes in the affected joint. ${ }^{[12]}$

Routine radiographs often are normal but may show bony erosion, especially if the hip is involved, well-defined erosions on both sides of a joint noted on plain radiographs signify advanced, diffuse disease. [20] [16]

MRI frequently is diagnostic, showing intraarticular masses that are low signal (dark) on the T1-weighted and T2-weighted images. Classically the difference in magnetic susceptibility between haemosiderin-laden PVNS and surrounding tissue may cause the lesion to appear larger (blooming) on gradient echo images. ${ }^{[19]}$

There is extensive overlap of the soft-tissue sarcoma appearances with MRI; the majority has a low signal $\mathrm{T} 1$ and heterogeneous high signal T2 appearances, exceptions to this may include high $\mathrm{T} 1$ signals in some liposarcomas and low T2 signal in desmoid fibromatosis and PVNS. The characteristics of particular tumors may be emphasized, such as a serpiginous pattern in vascular tumors, dystrophic calcification in synovial sarcomas or blooming artifact caused by the haemosiderin content of diffuse-type PVNS. [19]

MRI should be performed before biopsy as the tissue characteristics may alter. Contrast is reserved for targeting the biologically active areas of tumors for biopsy, although it risks nephrogenic systemic fibrosis in patients with chronic renal impairment. [19]

$M R I$ may show presence of fat signal within the lesion and MRI may reveal extra-articular extension of the process. Differential diagnosis includes reactive or inflammatory synovitis, hemophilia, or synovial chondromatosis. [16]

Additionally, the extent of the disease process may be further delineated with MRI. [20]

Recommended treatment for the localized form is marginal excision and for the diffuse form is total synovectomy. If there are significant secondary degenerative changes of the joint surfaces, arthroplasty should be strongly considered. [20]
For localized PVNS, simple excision of the diseased synovial tissue is considered the golden standard, ${ }^{[4]}$ and can achieve a $0 \%$ recurrence rate which the longest follow-up of 7 years. [5]

Surgical excision is the gold standard therapy to control symptoms and prevent further joint erosion, but high recurrence rates are related to incomplete excision. Joint erosion is particularly associated with the diffuse articular form, necessitating arthroplasty in the hip and the knee. [19]

Arthroscopic techniques are as effective as traditional open procedures if a complete synovectomy with multiple portals is performed. [17] Often an anterior arthroscopic synovectomy is combined with open posterior removal of any extra-articular disease. ${ }^{[16]}$

Arthroscopically assisted mini open synovectomy for the treatment of localized PVNS is also a safe alternative treatment style. However, Richter et al [6] reported that arthroscopic, open, and arthroscopically assisted mini open synovectomy had a high recurrence rate of $58 \%(69 / 118), 36 \%(35 / 97)$, and $50 \%(5 / 10)$, respectively.

Chin and Brick ${ }^{[7]}$ reported that the use of arthroscopic synovectomy in patients with extraarticular lesions of diffuse PVNS, and 38 patients had a poor prognosis with an average follow-up of 3.63 years. The cause of the high recurrence rate of arthroscopic treatment may be extensive joint involvement and extraarticular spread, and it is recommended that patients with diffuse PVNS should be treated with open synovectomy

Giant cell tumor of tendon sheath is treated with marginal excision. ${ }^{[16]}$

Hamlin et al. followed 18 patients at Mayo Clinic who were diagnosed with DPVNS, and underwent total knee arthroplasty, 14 with the diffuse type - active in 11 and inactive in three and four with the localized type. The mean follow-up was 9.9 years. All patients with the diffuse active type also underwent total synovectomy. At the end of the follow-up, in 14 of the 18 patients the replacement was fixed and 
with a satisfactory function. The four failures occurred in patients with the diffuse and active type of the disease. Three failures were due to aseptic loosening and one was due to relapse of the disease. These authors recommend a broad synovectomy, associated with posterior cruciate ligament replacement for better synovial membrane exposure. ${ }^{[15]}$

Adjunctive therapies include injection of intraarticular radiation materials and external beam radiotherapy. Radiotherapy in the diffuse lesion may be justified if surgery fails to control the process following multiple local recurrences. ${ }^{[19]}$ Lee et al ${ }^{[8]}$ analyzed that a total of 7 patients who were diagnosed as diffuse PVNS of the foot and ankle were treated with open synovectomy and adjuvant postoperative external radiotherapy, with a local control rate of up to $100 \%$ after followed up for 24 months.

Berger et al ${ }^{[9]}$ reported that 7 patients were treated with total dose of 30 to 50Gy postoperative radiotherapy, followed up for 29 months without recurrence and late radiation reactions. Horoschak et al ${ }^{[10]}$ believed that using 34 to $36 \mathrm{~Gy}$ radiation doses can achieve a better local control rate. Therefore, he suggested that $36 \mathrm{~Gy}$ be used as a conventional fractionated dose.

Alternative therapies such as tyrosine kinase inhibitors (e.g. Imatinib) have demonstrated efficacy in recurrent cases although the lesions tend to recur if the therapy stops. [19]
A 35-year-old previously healthy female presented with a 6-month right knee pain without history of trauma, the pain was mainly on posterior aspect and worsen by physical activity, it was progressive becoming sever enough to awaken her from sleep.

The pain was preceded by swelling in the posterior aspect of her knee for 2 years with increase in size in the last 6 months without change in skin color, she started to take NSAIDs but they had minimal effect on pain control, unremarkable personal \& family histories.

On examination, her right lower limb was well aligned, no pain was elicited by menisci and ligaments examination and stability tests were normal except for tenderness over the posterior aspect tenderness.

Range of Motion (ROM) was normal without restriction but she reported tenderness over infrapatellar region of the right knee with full flexion.

\section{First MRI Scan}

\section{Ultrasound Guided FNAC}

Ultrasound revealed a $\left(70^{*} 60^{*} 20\right) \mathrm{mm}$ mass in the popliteal fossa features in favor of synovial mass, under US guidance; FNA cytology reported large number of mixed inflammatory cells and hemosiderin-laden macrophages, multinu- cleated giant cells, and scattered spindle shape cells without malignant cells, Pathologist suggested a biopsy to confirm the diagnosis of PVNS

\section{Case Presentation}

Figure (1) T1 FSE Sagittal View MRI Scan

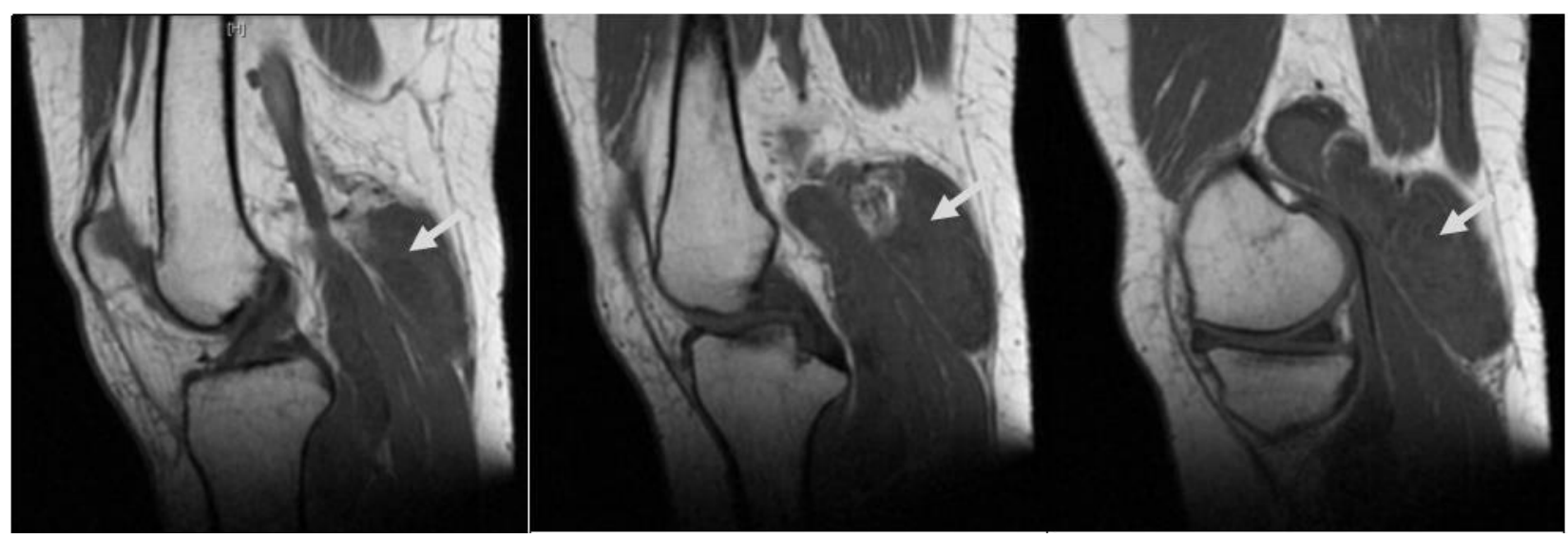

Low signal extra-articular lesion in posterior aspect of knee

IJCR: https://escipub.com/international-journal-of-case-reports/ 
Rebar Mohammed Noori et al., IJCR, 2021; 5:192

Figure (2) FSE Sagittal View MRI Scan

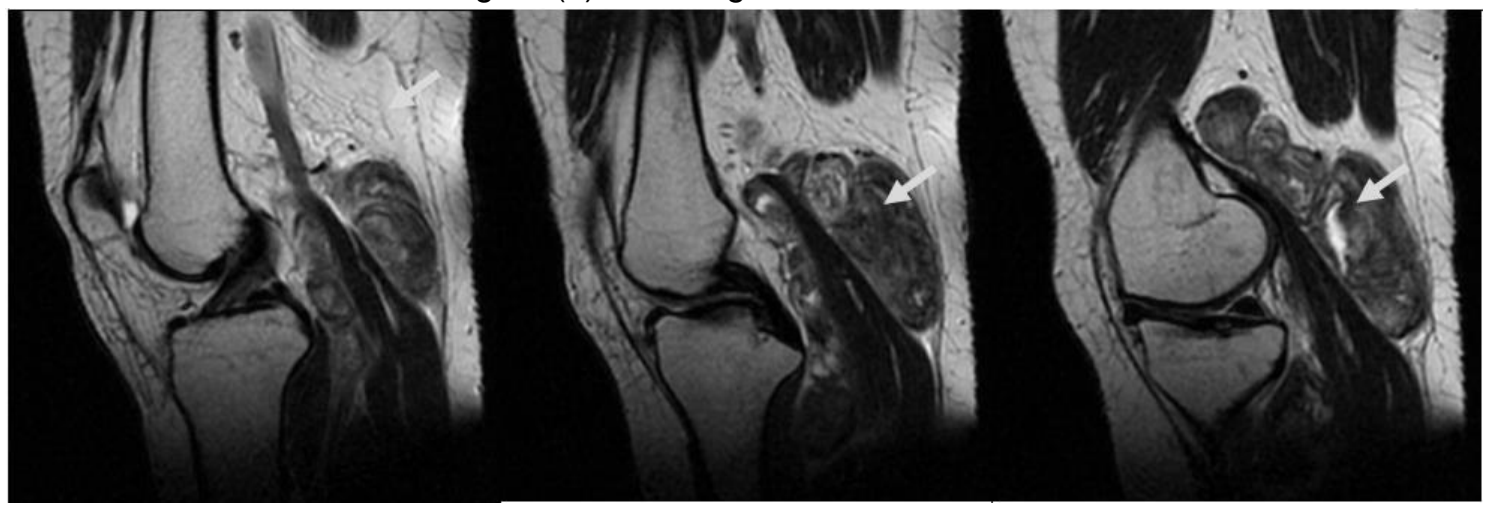

Heterogenous lobulated extra-articular lesion in posterior aspect of knee

Figure (3) PD FS Sagittal View MRI Scan

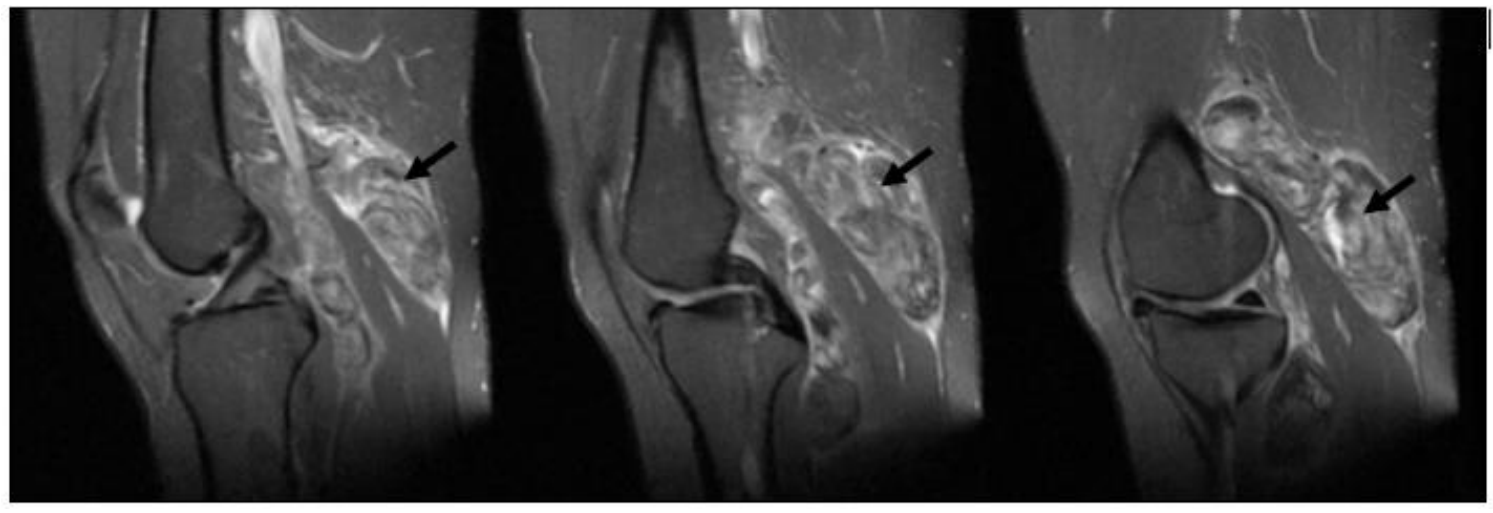

High signal heterogenous extra-articular lesion in posterior aspect of knee

Figure (4) PD FS Coronal View MRI Scan

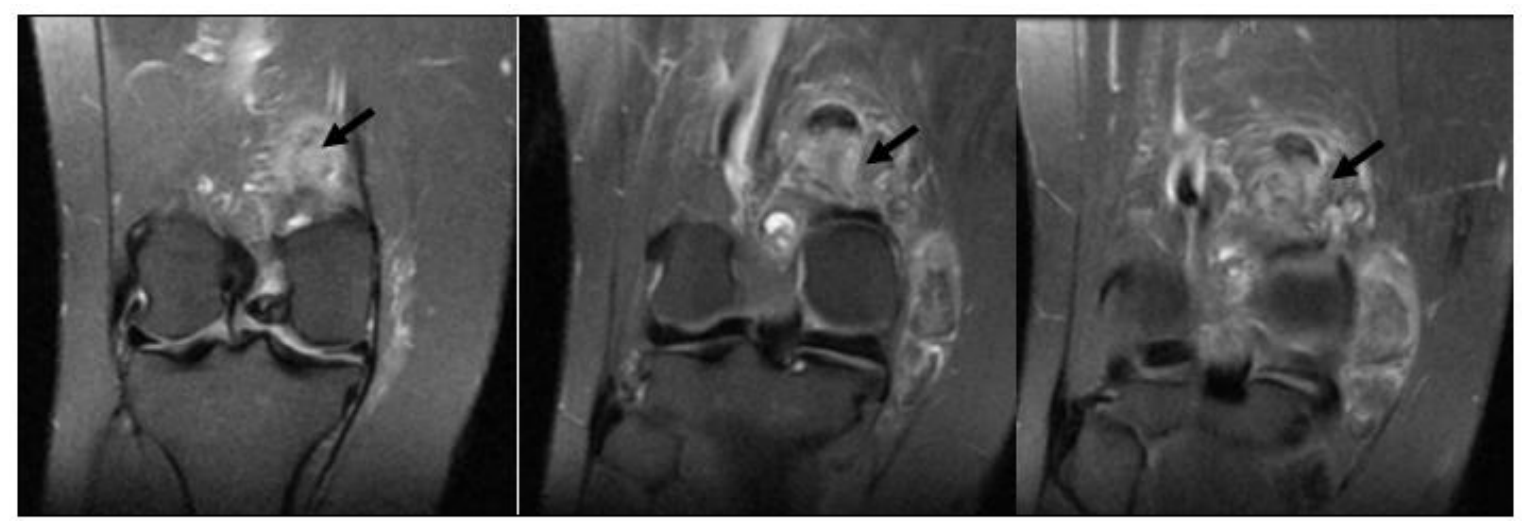

heterogenous extra-articular lesion in posterior aspect of knee

Figure (5) PD FS Axial View MRI Scan

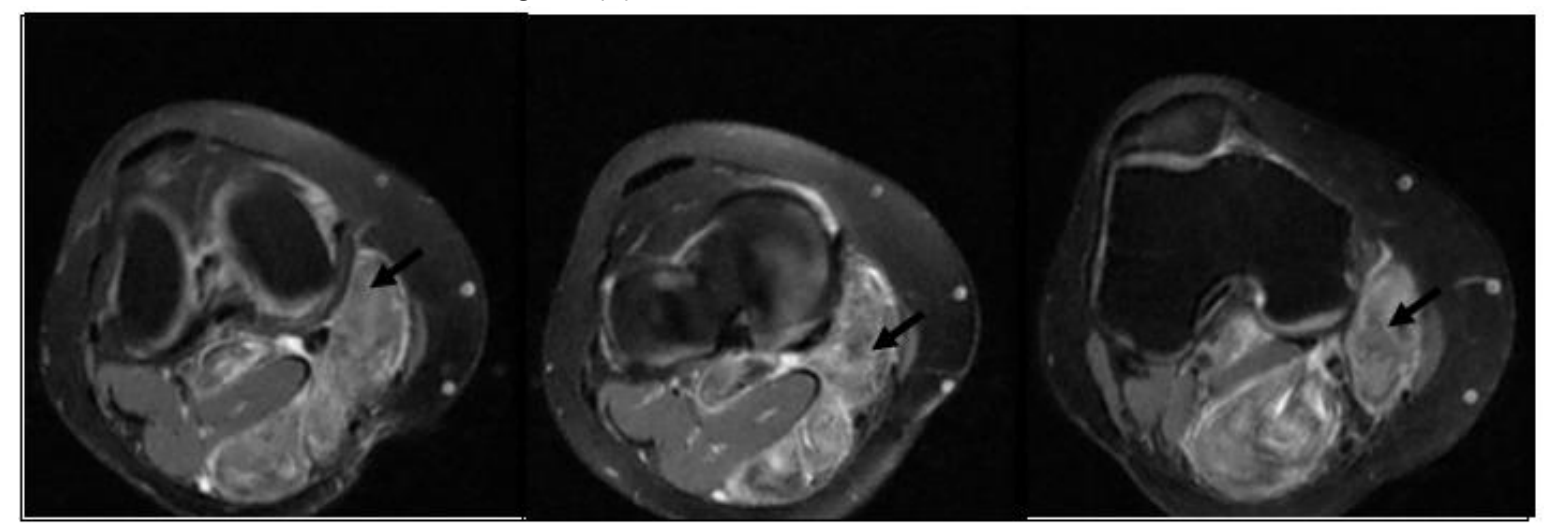

heterogenous extra-articular lesion in posterior aspect of knee IJCR: https://escipub.com/international-journal-of-case-reports/ 


\section{Tru Cut Biopsy}

Two tiny pieces of tissue measuring $0.3 \mathrm{~cm}$ in aggregate, brown in color, all had been taken in one block.

Serial sections revealed closely packed proliferated cells consisting of histiocytes and synovial cells admixed with large collection of hemosiderin laden macrophages, lipid laden macrophages (foamy cells) and fibroblastic elements, picture is highly suggestive of pigmented villonodular synovitis

\section{Surgical Procedure}

Under general anesthesia, diagnostic arthroscopy was done which showed normal exami- nation and normal synovial membrane, the patient then tuned to prone position, right knee was approached by S-shaped midline posterior approach.

Full thickness fascio-cutaneous flap was created, the medial gastrocnemius head was mobilized laterally, the lesion was exposed.

The lesion was lobulated brown-yellow color measuring around $15^{*} 8^{*} 5 \mathrm{~cm}$ and located medial to gastrocnemius muscle.

Total excision without exposure of the tumor was done and closure done primarily without drain, on the next day ambulation started with full weight bearing with crutch, flexion allowed as tolerated, postoperative period was uneventful. the specimen was sent for histopathological examination

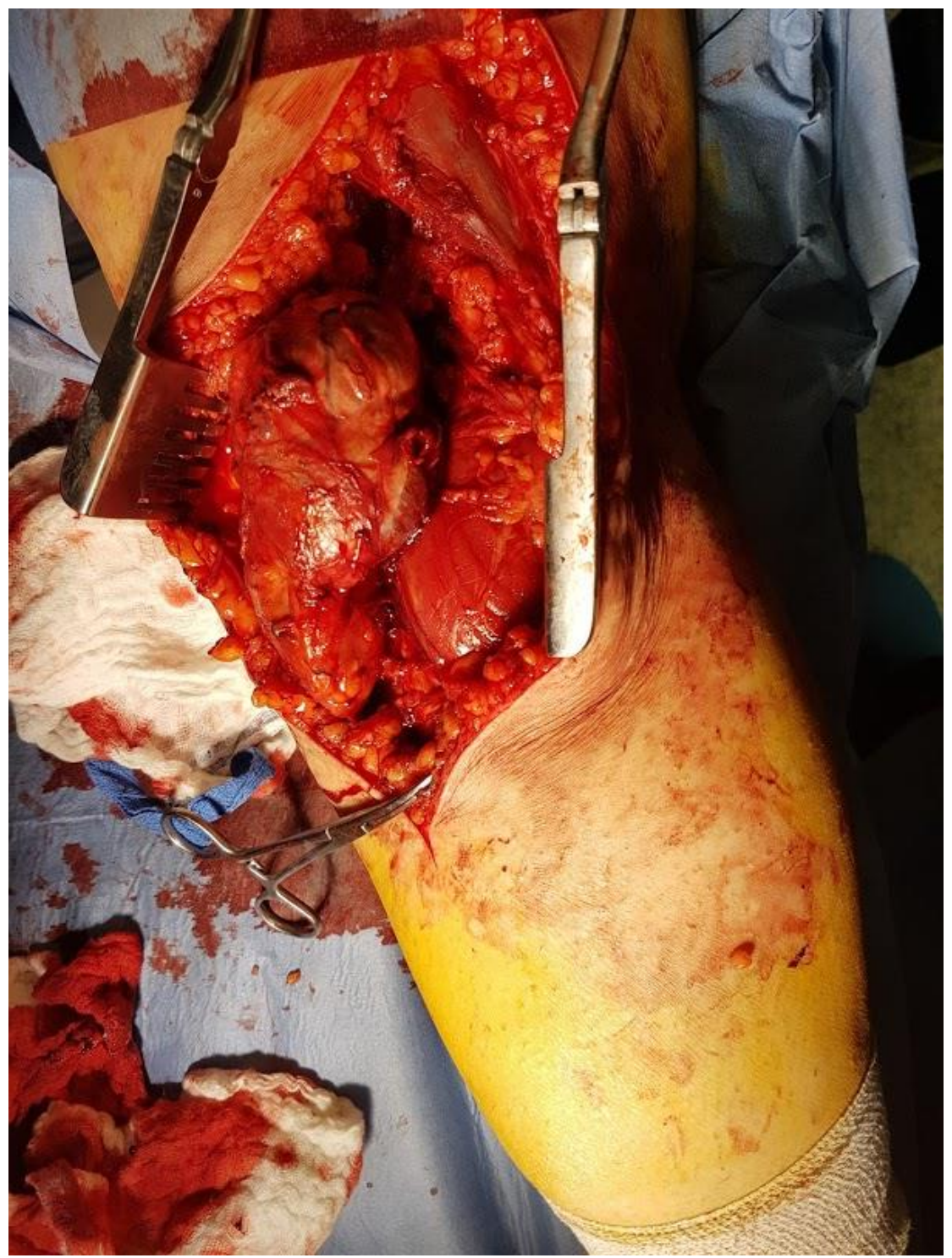

Figure (6) The lesion is located in posteromedial aspect of the right knee 
Rebar Mohammed Noori et al., IJCR, 2021; 5:192

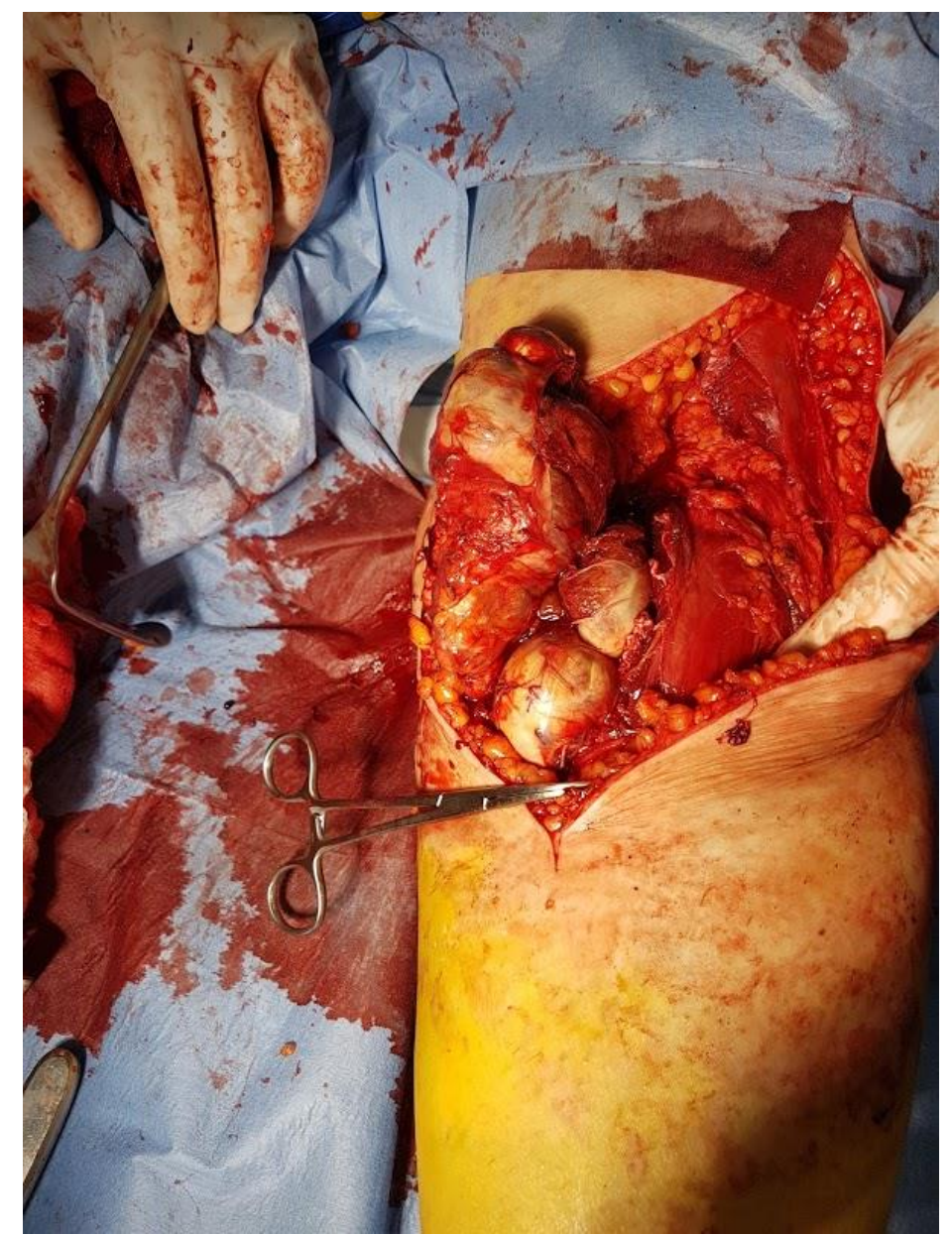

Figure (7) The lesion is lobulated and hemosiderin laden in color

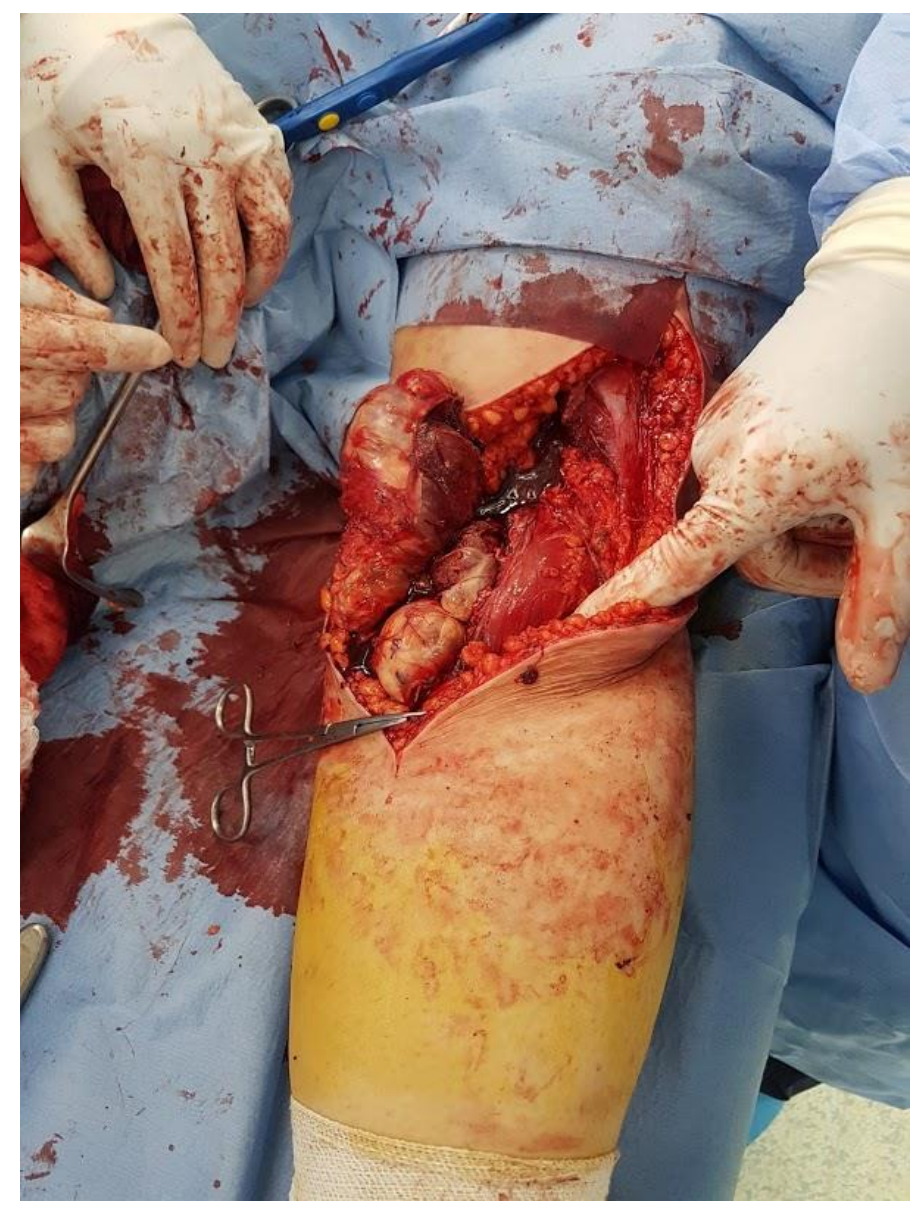

Figure (8) Lesion extension was approached for complete excision IJCR: https://escipub.com/international-journal-of-case-reports/ 


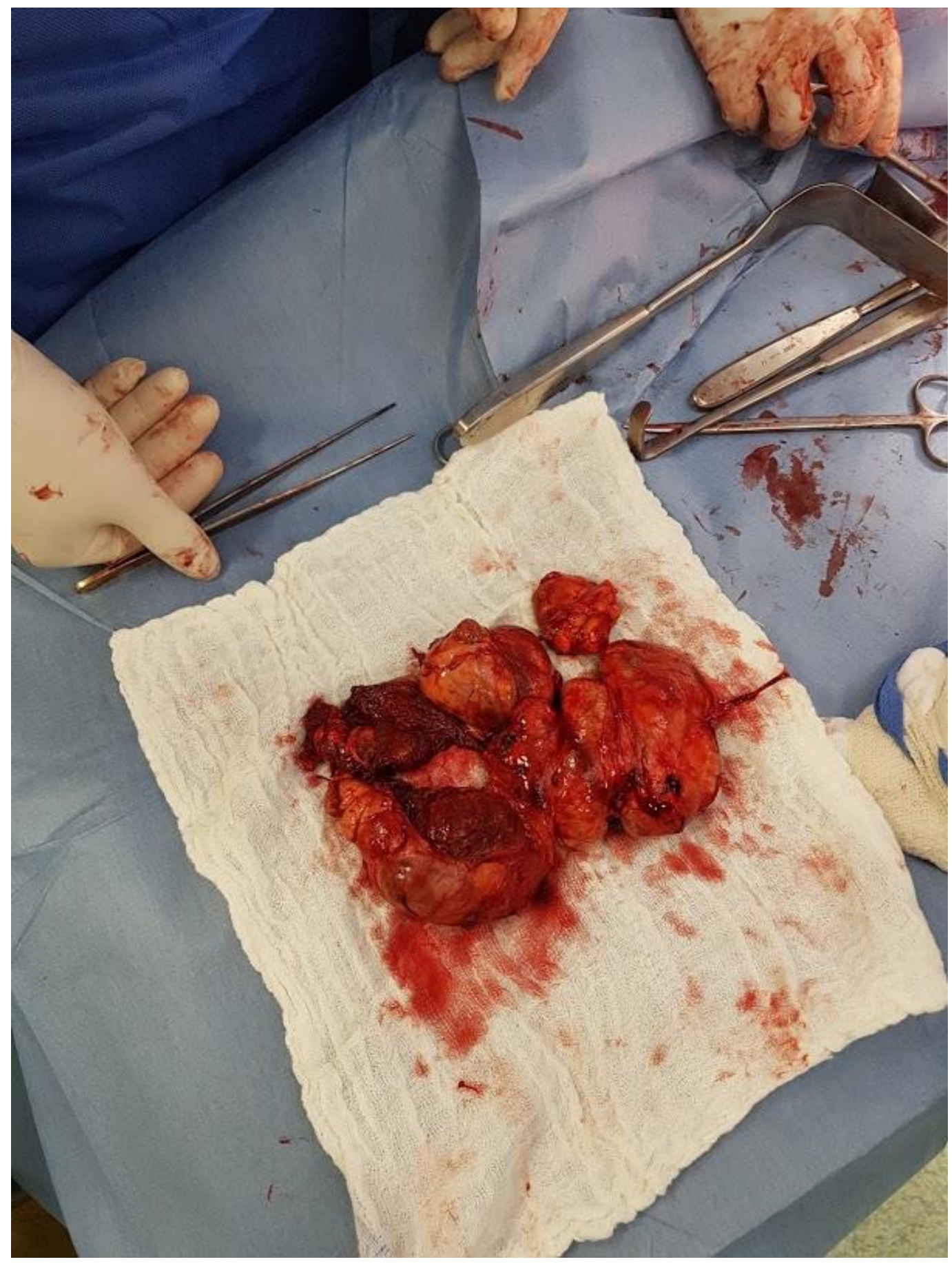

Figure (9) Lesion was excised completely

\section{Excisional Biopsy}

First pathologist reported papillary, villous and nodular structures showing proliferation of polygonal cells with eosinophillic cytoplasm, vesicular nucleoli; in a background of fibroconnective tissue which is covered by synovial lining abundant hemosiderin-laden and lipid laden macrophages are seen. Multinucleated giant cells are also present and presence of hyperplastic synovium with papillary projections; features suggestive of PVNS (nodular subtype). the proliferating polygonal cells show a degree of pleomorphisim with irregularity in nuclear membrane without mitotic count and necrosis.

\section{Recurrence}

pain was relieved for 1-year follow-up, then pain was started again in the same site of the first attack, gradually increasing in severity with mild swelling over the posterior aspect of the knee. Clinical examination was unremarkable apart from tenderness in the infrapatellar region with full flexion.

Active \& passive movements revealed full range of motion. 
Rebar Mohammed Noori et al., IJCR, 2021; 5:192

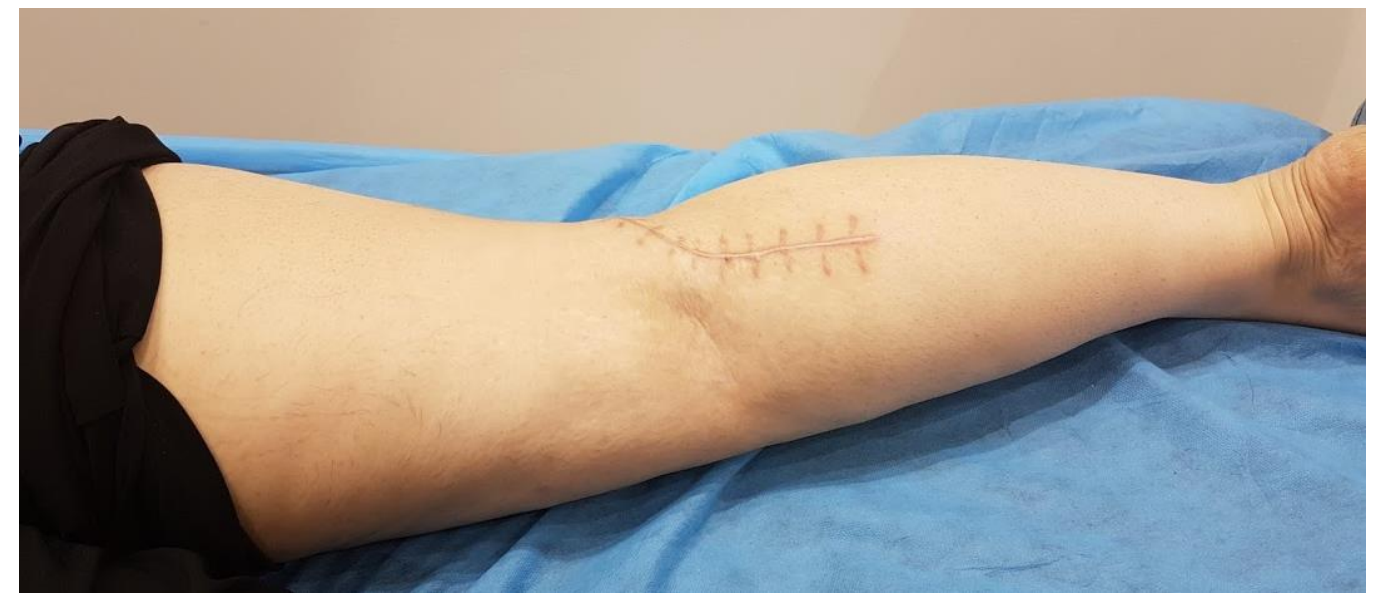

Figure (10) Full knee extension

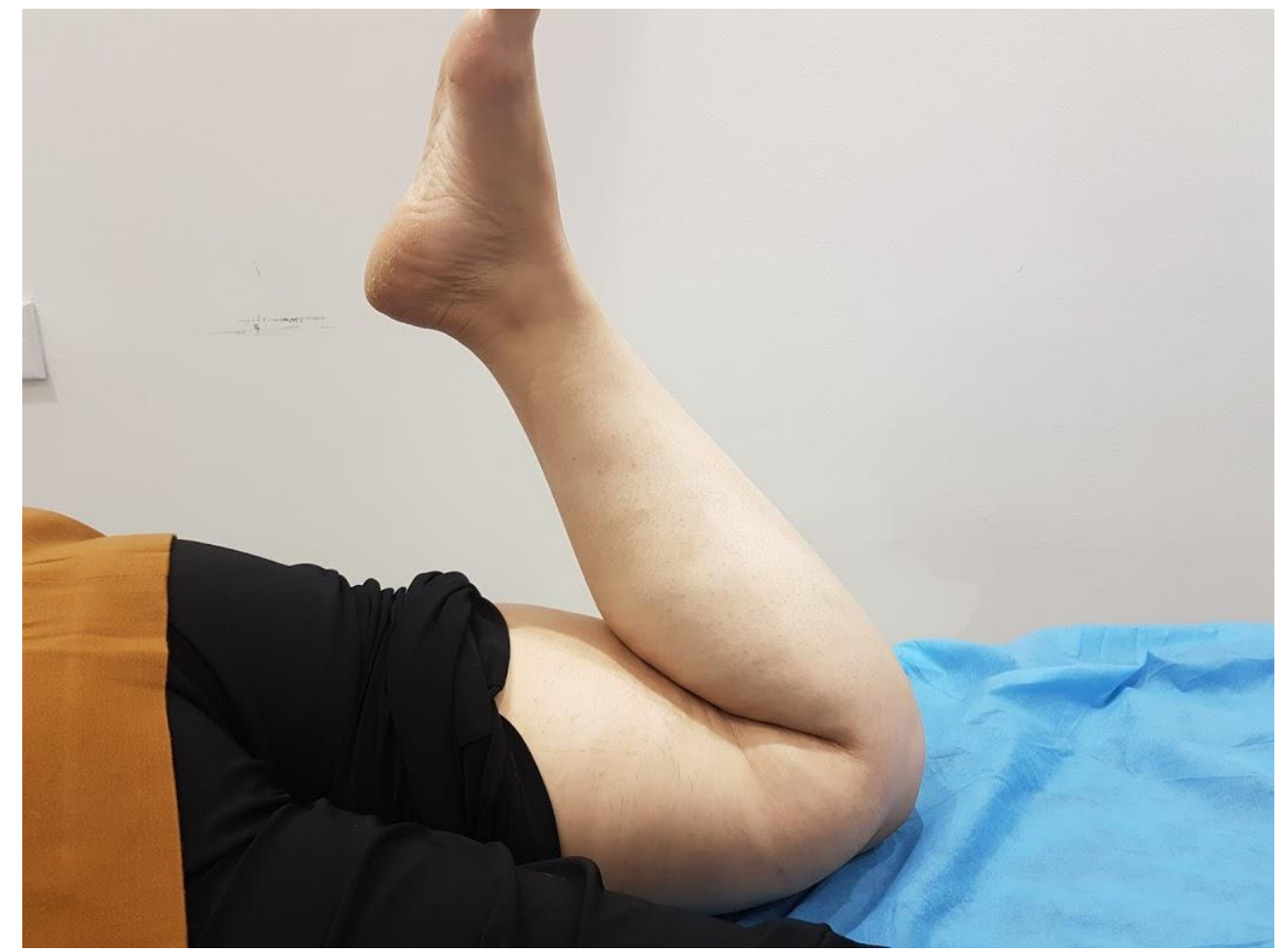

Figure (11) Full knee flexion

\section{Second MRI Scan}

Figure (12) T1 Sagittal View MRI Scan

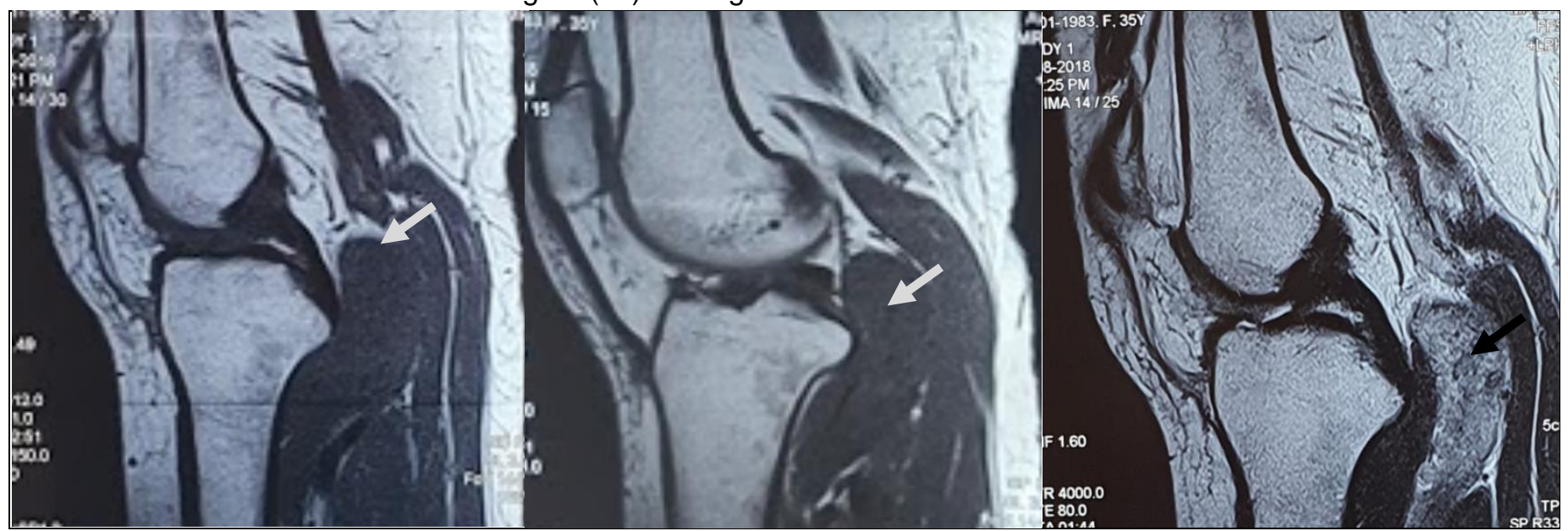

Low signal extra-articular lesion in posterior aspect of knee (white arrow), with longer TR \& TE MRI the lesion shows higher signal with more heterogeneity (black arrow) 
Figure (13) T2 Sagittal View MRI Scan

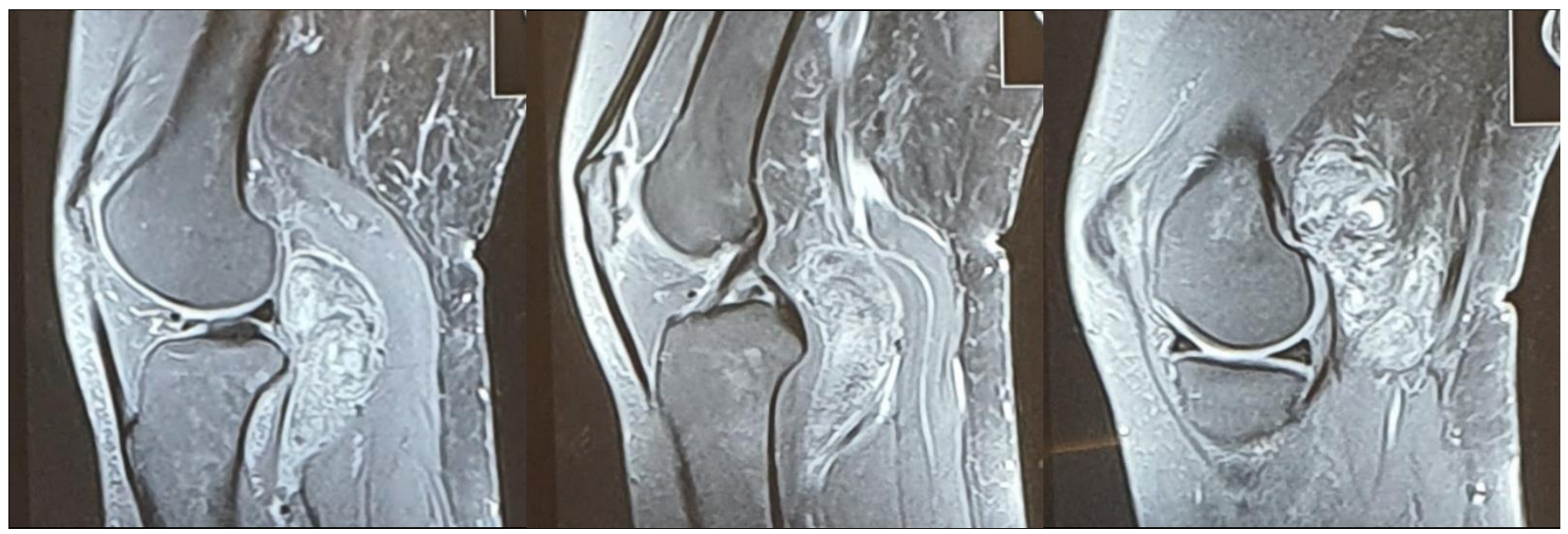

Well-defined heterogenous signal intensity lesion in posterior part of knee joint, extending around $2 \mathrm{~cm}$ above joint line and $4 \mathrm{~cm}$ below joint line near fibular head

Figure (14) T2 Coronal and Axial Views MRI Scan

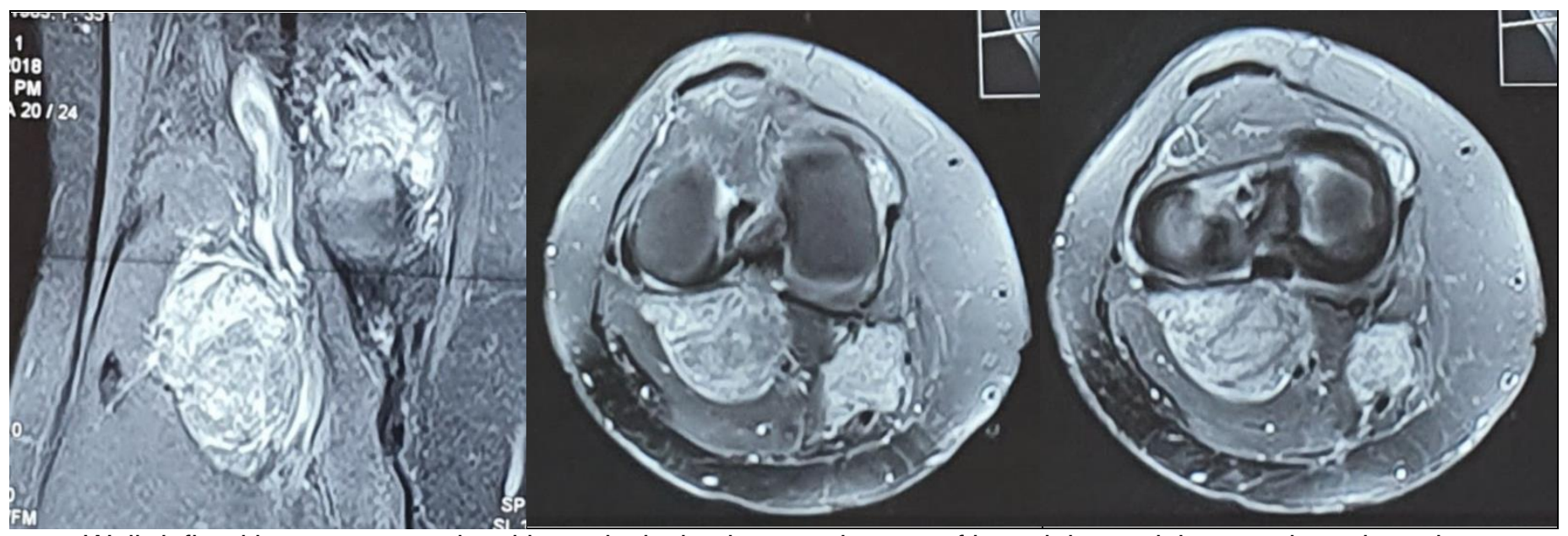

Well-defined heterogenous signal intensity lesion in posterior part of knee joint, mainly posterior to lateral femoral condyle without invasion to adjacent structures

\section{Knee Arthroscopy}

To tackle the recurrent lesion; 4-portal right knee arthroscopy was done.

Diffuse synovitis and oblique medial meniscal body tear were noted, the lesion was excised and the medial meniscus was trimmed.

\section{Discussion}

The incidence of PVNS is 1.8 new cases each year per million individuals [22], it is common in $4^{\text {th }} \& 5^{\text {th }}$ decades individuals ${ }^{[23]}$, some published studies described it as synovial giant cell, on the other hand it was described as localized pigmented villonodular synovitis by other studies [24]

While some authors suggested that synovial overgrowth may increase the pressure inside the involved joint which may cause bone erosion, others believed that bone erosion is caused by a substance released by synovium which may lead to joint destruction, still the mechanism of bone erosion is not clear [25].

PVNS has different possible etiology suggestions; repeated trauma, frequent hemarthrosis, benign neoplasm \& reactionary to unknown stimulus, so it has not established yet what causes this pathology [26].

While the diffuse form of PVNS may cause effusion and limitation in range of motion; localized form is difficult to diagnose with clinical features mimic meniscal tear or intra-articular loose body ${ }^{[27]}$. 
Clinical examination may reveal non-specific features as in our case; there was tenderness over the posterior aspect of the knee with neither effusion nor limitation in range of motion making intra-articular pathology a far possibility.

Our case first MRI images (Figures 1 till 5) showed a well-defined lobulated heterogenous lesion in posterior aspect of the right knee, the lesion is completely extra-articular but the signal intensity \& MRI images were suggestive of PVNS.

Tru-cut biopsy was compatible with MRI features of PVNS, being extra-articular PVNS is not uncommon, it was called pigmented villonodular tumor of tendon sheath or giant cell tumor of tendon sheath till it got its official term by WHO in 2013 as Tenosynovial Giant Cell Tumor.

But being extra-synovial is not common, to our knowledge; no reported cases of extra-synovial PVNS till this moment.

Based on MRI images \& Tru-cut biopsy results; an open surgical excision was planned, intraoperatively the lesion was big $\left(15^{*} 8 \mathrm{~cm}\right)$ well defined hemosiderin laden in color but not invading the adjacent gastrocnemius muscle (Figures 6 till 9).

Histopathological report of the excised lesion was confirming the diagnosis of Extra-synovial PVNS.

After 1-year, the patient developed gradual pain in the same area suggesting recurrence of the tumor, clinical examination revealed tenderness over the posterior aspect of the right knee with full range of motion (Figures 10 and 11).

Second MRI showed features suggestive of recurrence of the tumor (Figures 12 to 14) and second operation for excision was done by arthroscope, there was medial meniscal tear treated by meniscal trimming.

The aim of reporting this case is to expand our differential diagnosis list when we treat an extrasynovial lesion and opening a new opportunity to search deeper in etiology and pathophysiology of this tumor, Despite being a benign, there are reported cases of malignant transformation of $P$ VNS, but it is rare ${ }^{[28]}$.
In our case, the recurrence was after one year, this may hint to a higher recurrence rate and more rapid than conventional intra-synovial PVNS, we cannot assess recurrence rate by one reported case, a further research for recurrence rate of such lesions is recommended.

\section{Conclusion}

Pigmented Villonodular Synovitis can be intrasynovial and extra-synovial, by our case report of extra-synovial PVNS; we recommend that PVNS cannot be excluded in extra-synovial lesion assessment, surgical excision can improve patient symptoms but still the lesion can be recurrent.

\section{References}

[1] Ogilvie-Harris DJ, McLean J, Zarnett ME. Pigmented villonodular synovitis of the knee. The results of total arthroscopic synovectomy, partial, arthroscopic synovectomy, and arthroscopic local excision. J Bone Joint Surg Am 1992; 74:119-23.

[2] Nilsson M, Hoglund $M$, Panagopoulos I, et al. Molecular cytogenetic mapping of recurrent chromosomal breakpoints in tenosynovial giant cell tumors. Virchows Arch 2002;441:475-80.

[3] Dahlen A, Broberg K, Domanski HA, et al. Analysis of the distribution and frequency of trisomy 7 in vivo in synovia from patients with osteoarthritis and pigmented villonodular synovitis. Cancer Genet Cytogenet2001;131 :19-24.

[4] Hantes ME, Basdekis GK, Zibis $A H$, et al. Localized pigmented villonodular synovitis in the anteromedial compartment of the knee associated with cartilage lesions of the medial femoral condyle: report of a case and review of the literature. Knee Surg Sports Traumatol Arthrosc 2005; 13:209-12.

[5] Auregan JC, Bohu Y, Lefevre N, et al. Primary arthroscopic synovectomy for pigmented villonodular synovitis of the knee: recurrence rate and functional outcomes after a mean follow-up of seven years. Orthop Traumatol Surg Res 2013; 99:937-43.

[6] Richter M, Trzeciak T, Owecki M, et al. The role of adipocytokines in the pathogenesis of knee joint osteoarthritis. Int Orthopaed 2015; 39:1211 $-7$.

[7] Chin KR, Brick GW. Extraarticular pigmented villonodular synovitis: a cause for failed knee arthroscopy. Clin Orthop Relat Res 2002; 404: 330-8.

[8] Lee M, Mahroof S, Pringle J, et al. Diffuse pigm- 
ented villonodular synovitis of the foot and ankle treated with surgery and radiotherapy. Int Orthop 2005;29:403-5.

[9] Berger B, Ganswindt U, Bamberg $M$, et al. External beam radiotherapy as postoperative treatment of diffuse pigmented villonodular synovitis. Int J Radiat Oncol Biol Phys 2007; 67:1130-4.

[10] Horoschak M, Tran PT, Bachireddy P, et al. External beam radiation therapy enhances local control in pigmented villonodular synovitis. Int J Radiat Oncol Biol Phys 2009; 75:183-7.

[11] Myers B, Masi A, Feigenbaum S. Pigmented villonodular synovitis and tenosynovitis: a clinical epidemiologic study of 166 cases and literature review. Medicine (Baltimore). 1980; 59:223-238

[12] S. Clifton Willimon, Tim Schrader, Crystal A. Perkins, Arthroscopic Management of Pigmented Villonodular Synovitis of the Hip in Children and Adolescents. Orthop J Sports Med. 2018 Mar; 6(3): 2325967118763118.

[13] Oda Y., Takahira T., Yokoyama R., Tsuneyoshi M. Diffuse-type giant cell tumor/pigmented villonodular synovitis arising in the sacrum: malignant form. Pathol Int. 2007;57(9):627-631.

[14] Jaffe H.L., Lichtenstein L., Sutro C.J. Pigmented villonodular synovitis, bursitis and tenosynovitis. Arch Pathol. 1941; 31:731-765.

[15] Hamlin B.R., Duffy G.P., Trousdale R.T., Morrey B.F. Total knee arthroplasty in patients who have pigmented villonodular synovitis. J Bone Joint Surg Am. 1998;80(1):76-82.

[16] AAOS Comprehensive Orthopedic Review 2009, Volume 1, Chapter 4: Orthopedic Oncology and Systemic Disease, Page 454

[17] Miller's Review of Orthopaedics, $7^{\text {th }}$ Edition 2016, Chapter 4: Sport Medicine, Page 357
[18] Miller's Review of Orthopaedics, $7^{\text {th }}$ Edition 2016, Chapter 9: Orthopaedic Pathology, Page 717

[19] Apley \& Solomon's System of Orthopaedics and Trauma $10^{\text {th }}$ Edition 2018, Chapter 9: Tumors, Page 225

[20] Campbells Operative Orthopaedics $13^{\text {th }}$ edition 2017, Chapter 28: Soft Tissue Tumors, Page 990

[21] L. Pinheiro Junior, M. Cenni,R. Leal, and L. Teixeira, Total Knee Replacement in Patients with Diffuse Villonodular Synovitis, Rev Bras Ortop. 2017 Sep-Oct; 52(5): 616-620.

[22] Ushijima M, Hashimoto $H$, Tsuneyoshi M, Enjoji M. Pigmented villonodular synovitis. A clinicopathologic study of 52 cases. Acta Pathol Jpn. 1986;36(3):317-326

[23] Extraarticular pigmented villonodular synovitis: a cause for failed knee arthroscopy. Chin KR, Brick GW. Clin Orthop Relat Res. 2002; 404: 330-338.

[24] Abdalla JR, Cohen M, Nóbrega J, Forgas A. Tumor gigantocelular sinovial do joelho. Rev Bras Ortop. 2009;44(5):437-449.

[25] Pigmented villonodular synovitis: keys to early diagnosis. Bhimani MA, Wenz JF, Frassica FJ. Clin Orthop Relat Res. 2001; 386:197-202.

[26] Kim SJ, Shin SJ, Choi NH, Choo ET. Arthroscopic treatment for localized pigmented villonodular synovitis of the knee. Clin Orthop Relat Res. 2000;(379):330-379

[27] Aşik M, Erlap L, Altinel L, Cetik O. Localized pigmented villonodular synovitis of the knee. Arthroscopy. 2001;17

[28] Oda Y, Takahira T, Yokoyama R, Tsuneyoshi M. Diffuse-type giant cell tumor/pigmented villonodular synovitis arising in the sacrum: malignant form. Pathol Int. 2007;57(9):627-631.

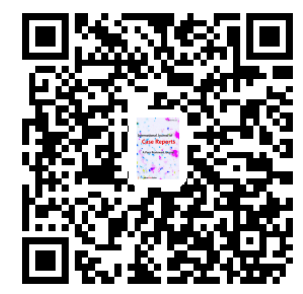

